\title{
Adiabatic elimination in quantum stochastic models
}

\author{
Luc Bouten and Andrew Silberfarb \\ Physical Measurement and Control 266-33, California Institute of Technology, \\ 1200 E. California Blvd., Pasadena, CA 91125, USA
}

\begin{abstract}
We consider a physical system with a coupling to bosonic reservoirs via a quantum stochastic differential equation. We study the limit of this model as the coupling strength tends to infinity. We show that in this limit the solution to the quantum stochastic differential equation converges strongly to the solution of a limit quantum stochastic differential equation. In the limiting dynamics the excited states are removed and the ground states couple directly to the reservoirs.
\end{abstract}

\section{Introduction}

It is a frequent occurence in physics to have a system that spends a very limited amount of time in its excited states. This is, for instance, the case if the system is strongly coupled to a low temperature environment (e.g. the optical field). The strong coupling ensures that excitations above the ground levels of the system quickly dissipate into its environment. It is therefore reasonable to ask for a model in which the excited states are eliminated from the description. That is, we would like to have a description that only involves the ground states of a system and its environment. The procedure for going from the full model to the reduced model is called adiabatic elimination.

We study adiabatic elimination in the context of quantum stochastic models [15] which arise by taking a weak coupling limit of QED (quantum electrodynamics) models 1 . 13, 5, and are widely applicable to systems studied in quantum optics. Specifically, quantum stochastic models are the starting point for deriving master equations, filtering equations, and input-output relations. In the quantum optics community adiabatic elimination is a common technique, used, for instance, in atomic systems [22, 2, 6, 11] and in cavity QED models [12, 23, as well as in more recent work on quantum feedback 7, 9, 24. Rigorous results have been demonstrated for adiabatic elimination outside of the quantum stochastic models we consider [19, 4, 11]. At present, however, apart from the work 14 on the elimination of a leaky cavity (using a Dyson series expansion to prove weak convergence), no rigorous results have been obtained on adiabatic elimination in the context of the quantum stochastic models introduced by Hudson and Parthasarathy [15. 
We start by considering a family, indexed by a parameter $k$, of quantum stochastic differential equations (QSDE's). The parameter $k$ can be interpreted as the coupling strength between the system and its environment. The environment is modelled by a collection of bosonic heat baths in the vacuum representation. We assume that the coefficients of the QSDE are all bounded and satisfy the usual conditions guaranteeing a unique unitary solution [15. We state further assumptions on the coefficients and show that under these assumptions the solution of the QSDE converges strongly to the solution of a limiting QSDE as $k$ tends to infinity (Theorem 2.1). The limiting QSDE represents the adiabatically eliminated time evolution of the system.

The heart of the proof is a technique introduced by T.G. Kurtz [17] that enables the application of the Trotter-Kato Theorem [21. This allows us to prove strong convergence of the unitaries using convergence of generators of semigroups rather than convergence of a Dyson series expansion. Convergence is first shown on the vacuum vector of the bosonic reservoirs. We then extend this result to any possible vector in the Hilbert space of the reservoirs by sandwiching the unitaries with Weyl operators and using a density argument.

The remainder of this article is organized as follows. In Section 2 we introduce the system coupled to $n$ bosonic reservoirs in the vacuum representation. We state assumptions on the coefficients of the QSDE and present the main convergence theorem. In Section 3 we discuss four applications of the theorem in the context of examples from atomic physics and cavity QED. Section 4 presents the proof of the main convergence theorem. In Section 5 we discuss our results.

\section{The main result}

Let $\mathcal{H}$ be a Hilbert space and let $n$ be an element of $\mathbb{N}$. Let $\mathcal{F}$ be the symmetric Fock space over $\mathbb{C}^{n} \otimes L^{2}\left(\mathbb{R}^{+}\right) \cong L^{2}\left(\mathbb{R}^{+} ; \mathbb{C}^{n}\right)$, i.e.

$$
\mathcal{F}=\mathbb{C} \oplus \bigoplus_{m=1}^{\infty} L^{2}\left(\mathbb{R}^{+} ; \mathbb{C}^{n}\right)^{\otimes_{s} m}
$$

Physically, the Hilbert space $\mathcal{H} \otimes \mathcal{F}$ describes a system $\mathcal{H}$ coupled to $n$ bosonic reservoirs (e.g. $n$ decay channels in the quantized electromagnetic field). For $f \in L^{2}\left(\mathbb{R}^{+} ; \mathbb{C}^{n}\right)$, we define the exponential vector $e(f)$ in $\mathcal{F}$ by

$$
e(f)=1 \oplus \bigoplus_{m=1}^{\infty} \frac{f^{\otimes m}}{\sqrt{m !}}
$$

Moreover, we define the coherent vector $\pi(f)$ to be the exponential vector $e(f)$ normalized to unity, i.e. $\pi(f)=\exp \left(-\frac{1}{2}\|f\|^{2}\right) e(f)$. The vacuum vector is defined to be the exponential vector $\Phi=e(0)=1 \oplus 0 \oplus 0 \ldots$ The expectation with respect to the vacuum vector is denoted by $\phi$, i.e. $\phi$ is a map from $\mathcal{B}(\mathcal{F})$ (the bounded operators on $\mathcal{F}$ ) to $\mathbb{C}$, given by $\phi(W)=\langle\Phi, W \Phi\rangle$ for all $W \in \mathcal{B}(\mathcal{F})$. 
The interaction between the system and the bosonic reservoirs is modelled by a quantum stochastic differential equation (QSDE) in the sense of Hudson and Parthasarathy [15] of the form

$$
d U_{t}^{(k)}=\left\{\left(S_{i j}^{(k)}-\delta_{i j}\right) d \Lambda_{t}^{i j}+L_{i}^{(k)} d A_{t}^{i \dagger}-L_{i}^{(k) \dagger} S_{i j}^{(k)} d A_{t}^{j}+K^{(k)} d t\right\} U_{t}^{(k)},
$$

where $U_{0}^{(k)}=I$. We consistently use the convention that repeated indices that are not within parentheses are being summed ( $i$ and $j$ run through $\{1, \ldots, n\})$. The Hilbert space adjoint is denoted by a dagger ${ }^{\dagger}$. We have indexed the equation with a positive number $k$, and in the following we will be interested in the behaviour of $U_{t}^{(k)}$ as $k$ tends to infinity. We assume that the following conditions on the coefficients of the QSDE are satisfied.

Assumption 1: For each $k \geq 0$, the coefficients $K^{(k)}, S_{i j}^{(k)}$ and $L_{i}^{(k)}(i, j \in\{1, \ldots, n\})$ of the quantum stochastic differential equation (11) are bounded operators on $\mathcal{H}$. Furthermore, for each $k \geq 0$, the coefficients satisfy the following relations

$$
K^{(k)}+K^{(k) \dagger}=-L_{i}^{(k) \dagger} L_{i}^{(k)}, \quad S_{i l}^{(k)} S_{j l}^{(k) \dagger}=\delta_{i j} I, \quad S_{l i}^{(k) \dagger} S_{l j}^{(k)}=\delta_{i j} I .
$$

Hudson and Parthasarathy [15] show that under Assumption 1, the quantum stochastic differential equation (1) has a unique unitary solution $U_{t}^{(k)}$, and, the adjoint $U_{t}^{(k) \dagger}$ satisfies the adjoint of Eq. (1).

Assumption 2: There exist bounded operators $Y, A, B, F_{i}, G_{i}$ and $W_{i j}$ (independent of $k$ ) on $\mathcal{H}$ such that

$$
K^{(k)}=k^{2} Y+k A+B, \quad L_{i}^{(k)}=k F_{i}+G_{i}, \quad S_{i j}^{(k)}=W_{i j},
$$

for all $i, j \in\{1, \ldots, n\}$.

We define $P_{0}$ as the orthogonal projection onto $\operatorname{Ker}(Y)$. Let $P_{1}=I-P_{0}$ be its complement in $\mathcal{H}$. We use the following notation $\mathcal{H}_{0}=P_{0} \mathcal{H}$ and $\mathcal{H}_{1}=P_{1} \mathcal{H}$. Physically, one should think of $\mathcal{H}_{0}$ as the ground states and of $\mathcal{H}_{1}$ as the excited states of the system.

Assumption 3: There exists a bounded operator $Y_{1}^{-1}$ on $\mathcal{H}$ such that $P_{1} Y_{1}^{-1}=Y_{1}^{-1} P_{1}$ and

$$
Y Y_{1}^{-1} P_{1} Z P_{0}=P_{1} Z P_{0}, \quad P_{0} X P_{1} Y_{1}^{-1} Y=P_{0} X P_{1},
$$

where $Z=A, F_{i}^{\dagger} W_{i j},(j \in\{1, \ldots, n\})$ and $X=A, B, F_{i}, G_{i}, W_{i j}, G_{i}^{\dagger} W_{i j}, F_{i} Y_{1}^{-1} F_{j}$, $F_{i} Y_{1}^{-1} A, F_{i} Y_{1}^{-1} F_{l}^{\dagger} W_{l j}, A Y_{1}^{-1} A, A Y_{1}^{-1} F_{i}, A Y_{1}^{-1} F_{l}^{\dagger} W_{l j},(i, j \in\{1, \ldots, n\})$. Moreover, for all $i, j \in\{1, \ldots, n\}$ the following products are zero

$$
P_{0} Y P_{1}=P_{0} A P_{0}=F_{i} P_{0}=P_{0}\left(\delta_{i l}+F_{i} Y_{1}^{-1} F_{l}^{\dagger}\right) W_{l j} P_{1}=0 .
$$


Note that the existence of $Y_{1}^{-1}$ satisfying the assumptions in Eq. (2) is immediate if $Y$ maps $\mathcal{H}_{1}$ surjectively onto $\mathcal{H}_{1}$ and is therefore invertible on $\mathcal{H}_{1}$.

Definition 1: Suppose Assumption 2 and 3 hold. We define for all $i, j \in\{1, \ldots, n\}$ the following bounded operators on $\mathcal{H}$

$$
\begin{aligned}
& K=P_{0}\left(B-A Y_{1}^{-1} A\right) P_{0}, \quad L_{i}=\left(G_{i}-F_{i} Y_{1}^{-1} A\right) P_{0}, \\
& S_{i j}=\left(\delta_{i l}+F_{i} Y_{1}^{-1} F_{l}^{\dagger}\right) W_{l j} P_{0} .
\end{aligned}
$$

Assumption 4: For all $i, j \in\{1, \ldots, n\}$ the following products are zero

$$
P_{1} L_{i}=P_{1} S_{i j}=0 .
$$

Lemma 1: Suppose that Assumption 1, 2, 3 and 4 hold. The operators in Definition 1 satisfy

$$
K+K^{\dagger}=-L_{i}^{\dagger} L_{i}, \quad S_{i l} S_{j l}^{\dagger}=\delta_{i j} P_{0}, \quad S_{l i}^{\dagger} S_{l j}=\delta_{i j} P_{0} .
$$

Proof. By Assumptions 1 and2 we have $K^{(k)}+K^{(k)}=-L_{i}^{(k) \dagger} L_{i}^{(k)}, K^{(k)}=k^{2} Y+k A+B$ and $L_{i}^{(k)}=k F_{i}+G_{i}$ for all $k \geq 0$. Moreover, $F_{i} P_{0}=0$, by Assumption 3. Combining these results leads to

$$
\begin{aligned}
& -F_{i}^{\dagger} F_{i}=Y+Y^{\dagger} \\
& -P_{1} F_{i}^{\dagger} G_{i} P_{0}=P_{1}\left(A+A^{\dagger}\right) P_{0} \\
& -P_{0} G_{i}^{\dagger} G_{i} P_{0}=P_{0}\left(B+B^{\dagger}\right) P_{0} .
\end{aligned}
$$

We then use $Y Y_{1}^{-1} A P_{0}=A P_{0}$ from Assumption 3 and $L_{i}$ from Definition 1 to derive

$$
\begin{aligned}
-L_{i}^{\dagger} L_{i}= & -P_{0}\left(G_{i}^{\dagger}-A^{\dagger} Y_{1}^{-1 \dagger} F_{i}^{\dagger}\right)\left(G_{i}-F_{i} Y_{1}^{-1} A\right) P_{0} \\
= & P_{0}\left(B+B^{\dagger}\right) P_{0}-P_{0} A^{\dagger} Y_{1}^{-1 \dagger}\left(A+A^{\dagger}\right) P_{0} \\
& -P_{0}\left(A+A^{\dagger}\right) Y_{1}^{-1} A P_{0}+P_{0} A^{\dagger}\left(Y_{1}^{-1 \dagger}+Y_{1}^{-1}\right) A P_{0} \\
= & P_{0}\left(B+B^{\dagger}\right) P_{0}-P_{0} A Y_{1}^{-1} A P_{0}-P_{0} A^{\dagger} Y_{1}^{-1 \dagger} A^{\dagger} P_{0} \\
= & P_{0}\left(K+K^{\dagger}\right) P_{0} .
\end{aligned}
$$

By Definition 1

$$
S_{i j}=\left(\delta_{i l}+F_{i} Y_{1}^{-1} F_{l}^{\dagger}\right) W_{l j} P_{0} .
$$

Combining this with $-F_{i}^{\dagger} F_{i}=Y+Y^{\dagger}$ from above,

$$
\begin{aligned}
S_{l i}^{\dagger} S_{l j} & =P_{0} W_{m i}^{\dagger}\left(\delta_{m l}+F_{m} Y_{1}^{-1 \dagger} F_{l}^{\dagger}\right)\left(\delta_{l n}+F_{l} Y_{1}^{-1} F_{n}^{\dagger}\right) W_{n j} P_{0} \\
& =P_{0} W_{l i}^{\dagger} W_{l j} P_{0}=P_{0} \delta_{i j} .
\end{aligned}
$$


Then use $P_{0}\left(\delta_{i l}+F_{i} Y_{1}^{-1} F_{l}^{\dagger}\right) W_{l j} P_{1}=0$ from Assumption 3 and $P_{1} S_{i j} P_{0}=0$ from Asumption 4 to derive

$$
\begin{aligned}
S_{i l} S_{j l}^{\dagger} & =P_{0}\left(\delta_{i n}+F_{i} Y_{1}^{-1} F_{n}^{\dagger}\right) W_{n l} W_{m l}^{\dagger}\left(\delta_{m j}+F_{m} Y_{1}^{-1 \dagger} F_{j}^{\dagger}\right) P_{0} \\
& =P_{0}\left(\delta_{i n}+F_{i} Y_{1}^{-1} F_{n}^{\dagger}\right)\left(\delta_{n j}+F_{n} Y_{1}^{-1 \dagger} F_{j}^{\dagger}\right) P_{0}=\delta_{i j} P_{0} .
\end{aligned}
$$

The operators given by Definition 1 are the coefficients of a QSDE on the Hilbert space $\mathcal{H} \otimes \mathcal{F}$

$$
d U_{t}=\left\{\left(S_{i j}-\delta_{i j} P_{0}\right) d \Lambda_{t}^{i j}+L_{i} d A_{t}^{i \dagger}-L_{i}^{\dagger} S_{i j} d A_{t}^{j}+K d t\right\} U_{t}, \quad U_{0}=I .
$$

Lemma 1 implies that under Assumptions 1, 2, 3 and 4, Eq. (4) has a unique unitary solution on $\mathcal{H}$ [15], and, the adjoint $U_{t}^{\dagger}$ satisfies the adjoint of Eq. (44). Moreover, $U_{t}$ maps $\mathcal{H}_{0}$ to $\mathcal{H}_{0}$. Note that $U_{t} P_{1}=P_{1}$.

Theorem 2.1: Suppose Assumption 1, 2, 3 and 4 hold. Let $U_{t}^{(k)}$ be the unique unitary solution to Eq. (11). Let $U_{t}$ be the unique unitary solution to Eq. (4) where the coefficients are given by Definition 1, Then $U_{t}^{(k)} P_{0}$ converges strongly to $U_{t} P_{0}$, i.e.

$$
\lim _{k \rightarrow \infty} U_{t}^{(k)} \psi=U_{t} \psi, \quad \forall \psi \in \mathcal{H}_{0} \otimes \mathcal{F} .
$$

We prove Theorem 2.1 in Section 4

\section{Examples}

We use the following definitions in the first two examples below. Let $(|e\rangle,|g\rangle)$ be an orthogonal basis of $\mathbb{C}^{2}$. Define the raising and lowering operators in this basis as

$$
\sigma_{+}=\left(\begin{array}{cc}
0 & 1 \\
0 & 0
\end{array}\right), \quad \sigma_{-}=\left(\begin{array}{ll}
0 & 0 \\
1 & 0
\end{array}\right) .
$$

Define the Pauli operators

$$
\sigma_{x}=\sigma_{+}+\sigma_{-}, \quad \sigma_{y}=-i \sigma_{+}+i \sigma_{-}, \quad \sigma_{z}=\sigma_{+} \sigma_{-}-\sigma_{-} \sigma_{+},
$$

and define the projectors

$$
P_{e}=\sigma_{+} \sigma_{-}, \quad P_{g}=\sigma_{-} \sigma_{+}
$$


Example 1: (A two-level atom driven by a laser) The Hilbert space for a two-level atom is $\mathcal{H}=\mathbb{C}^{2}$, with $|e\rangle$ the excited state, and $|g\rangle$ the ground state. Define the detuning $\Delta \in \mathbb{R}$, the decay rate $\gamma \geq 0$ and the complex amplitude $\alpha \in \mathbb{C}$. The QSDE for this system in the electric dipole and rotating wave approximations is [2]

$$
\begin{aligned}
d U_{t}^{(k)}=\{ & k \sqrt{\gamma} \sigma_{-} d A_{t}^{\dagger}-k \sqrt{\gamma} \sigma_{+} d A_{t}-i k \alpha \sigma_{+} d t-i k \bar{\alpha} \sigma_{-} d t \\
& \left.-\frac{k^{2} \gamma}{2} \sigma_{+} \sigma_{-} d t-i k^{2} \Delta \sigma_{+} \sigma_{-} d t\right\} U_{t}^{(k)}, \quad U_{0}^{(k)}=I .
\end{aligned}
$$

Define the operators $Y, A, B, F, G, W$ as

$$
\begin{array}{rlrl}
Y & =(-i \Delta-\gamma / 2) \sigma_{+} \sigma_{-}, \quad A & =-i \alpha \sigma_{+}-i \alpha \sigma_{-}, \quad B=0, \\
F & =\sqrt{\gamma} \sigma_{-}, \quad G=0, \quad W=I .
\end{array}
$$

This satisfies Assumptions 1 and 2 and $P_{0}=P_{g}$. We take $Y_{1}^{-1}=-(i \Delta+\gamma / 2)^{-1} \sigma_{+} \sigma_{-}$, and Assumption 3 holds by inspection. Definition 1 leads to the following coefficients

$$
K=-\frac{|\alpha|^{2}}{i \Delta+\gamma / 2} P_{g}, \quad L=-i \frac{\alpha \sqrt{\gamma}}{i \Delta+\gamma / 2} P_{g}, \quad S=\frac{i \Delta-\gamma / 2}{i \Delta+\gamma / 2} P_{g} .
$$

Note that $P_{1} L=P_{1} S=0$ satisfying Assumption 4 . Theorem 2.1 then shows that $U_{t}^{(k)} P_{0}$ converges strongly to $U_{t} P_{0}$, given by

$$
d U_{t}=\frac{P_{g}}{i \Delta+\gamma / 2}\left\{-\gamma d \Lambda_{t}-i \alpha \sqrt{\gamma} d A_{t}^{\dagger}+i \bar{\alpha} \sqrt{\gamma} d A_{t}-|\alpha|^{2} d t\right\} U_{t}, \quad U_{0}=I .
$$

In the case that $\gamma=0$ the two level atom decouples from the field. In this case we may explicitly calculate the ground state evolution as

$$
P_{0} e^{-i\left(k \alpha \sigma_{+}+k \bar{\alpha} \sigma_{-}+k^{2} \Delta \sigma_{+} \sigma_{-}\right) t} P_{0}=\frac{e^{-i k^{2} \Delta t / 2}}{\Omega}(\Omega \cos (k \Omega t)+i k \Delta \sin (k \Omega t)),
$$

with $\Omega=\sqrt{\Delta^{2} k^{2}+4|\alpha|^{2}}$. For $k \rightarrow \infty$ this expression limits to $e^{i|\alpha|^{2} / \Delta}$ which is the solution to our eliminated differential equation $d U_{t}=i \frac{|\alpha|^{2}}{\Delta} U_{t} d t, U_{0}=I$.

Example 2: (Alkali atom) Now consider a system with Hilbert space $\mathcal{H}=\mathbb{C}^{2} \otimes \mathbb{C}^{2}$. Physically, the system represents an alkali atom with no nuclear spin coupled to a driving field on the $S_{1 / 2} \rightarrow P_{1 / 2}$ transition. We have four orthogonal states in this system corresponding to the atomic excited and ground states with angular momentum $m_{z}= \pm \frac{1}{2}$ along the $z$-axis. We define a detuning $\Delta \in \mathbb{R}$, a decay rate $\gamma \geq 0$ and a magnetic field $B_{i} \in \mathbb{R}, i \in x, y, z$. The system may emit into $n=3$ independent dipole modes, $A_{t}^{i}$, where the modes are labelled by $i \in\{x, y, z\}$. The QSDE for this system in the dipole and rotating wave approximations is [2],

$$
\begin{aligned}
d U_{t}^{(k)}=\{ & k \sqrt{\gamma} \sigma_{-} \otimes \sigma_{i} d A_{t}^{i \dagger}-k \sqrt{\gamma} \sigma_{+} \otimes \sigma_{i} d A_{t}^{i}-\frac{3 k^{2} \gamma}{2} P_{e} \otimes I d t \\
& \left.-i\left(k^{2} \Delta P_{e} \otimes I+I \otimes B_{i} \sigma_{i}\right) d t\right\} U_{t}^{(k)}, \quad U_{0}^{(k)}=I .
\end{aligned}
$$


Defining the operators $Y, A, B, F_{i}, G_{i}, W_{i j}$ as

$$
\begin{aligned}
& Y=\left(-i \Delta-\frac{3 \gamma}{2}\right) P_{e} \otimes I, \quad A=0, \quad B=-i I \otimes B_{i} \sigma_{i} \\
& F_{i}=\sqrt{\gamma} \sigma_{-} \otimes \sigma_{i}, \quad G_{i}=0, \quad W_{i j}=\delta_{i j},
\end{aligned}
$$

satisfies Assumptions 1 and 2, and $P_{0}=P_{g} \otimes I$. We take $Y_{1}^{-1}=-\left(i \Delta+\frac{3 \gamma}{2}\right)^{-1} P_{e} \otimes I$, and Assumption 3 holds by inspection. Define the eliminated coefficients as

$$
K=-i P_{g} \otimes B_{i} \sigma_{i}, \quad L_{i}=0, \quad S_{i j}=P_{g} \otimes\left(\delta_{i j} I-\frac{\gamma}{i \Delta+\frac{3 \gamma}{2}} \sigma_{i} \sigma_{j}\right) .
$$

This satisfies Assumption 4. Theorem 2.1 then shows that $U_{t}^{(k)} P_{0}$ converges strongly to $U_{t} P_{0}$, given by

$$
d U_{t}=P_{g} \otimes\left\{-i B_{i} \sigma_{i} d t-\frac{\gamma}{i \Delta+\frac{3 \gamma}{2}} \sigma_{i} \sigma_{j} d \Lambda_{t}^{i j}\right\} U_{t}, \quad U_{0}=I .
$$

In the following two examples we make use of a truncated harmonic oscillator. We have truncated the oscillator to satisfy the boundedness condition of Assumption 1 in the following two examples. Let $N$ be an element in $\mathbb{N}$ such that $N \geq 2$. The Hilbert space of the oscillator is $\mathbb{C}^{N}$. We choose an orthonormal basis $(|0\rangle, \ldots,|N-1\rangle)$ in $\mathbb{C}^{N}$. The annihilation operator $b: \mathbb{C}^{N} \rightarrow \mathbb{C}^{N}$ is given by

$$
b|n\rangle=\sqrt{n}|n-1\rangle, \quad n \in\{1, \ldots, N-1\},
$$

and $b|0\rangle=0$. The creation operator is defined to be the adjoint $b^{\dagger}$.

Example 3: (Gough and Van Handel [14]) Let $\mathfrak{h}$ be a Hilbert space. We define $\mathcal{H}=\mathfrak{h} \otimes \mathbb{C}^{N}$. The Hilbert space $\mathfrak{h}$ describes a system inside a cavity. We model the cavity as a truncated oscillator $\mathbb{C}^{N}$. Let $E_{i j}, i, j \in\{0,1\}$ be bounded operators on $\mathfrak{h}$ such that $E_{i j}^{\dagger}=E_{j i}$ and $\left\|E_{11}\right\|<\frac{\gamma}{2}$. Consider the following QSDE

$$
d U_{t}^{(k)}=\left\{\sqrt{\gamma} k b d A_{t}^{\dagger}-\sqrt{\gamma} k b^{\dagger} d A_{t}-\frac{\gamma k^{2}}{2} b^{\dagger} b d t-i H^{(k)} d t\right\} U_{t}^{(k)}, \quad U_{0}^{(k)}=I .
$$

Here $\gamma$ is a real parameter and $H^{(k)}$ is given by

$$
H^{(k)}=k^{2} E_{11} b^{\dagger} b+k E_{10} b^{\dagger}+k E_{01} b+E_{00} .
$$

Define operators $Y, A, B, F, G, W$ as

$$
\begin{aligned}
& Y=\left(-i E_{11}-\frac{\gamma}{2}\right) b^{\dagger} b, \quad A=-i\left(E_{10} b^{\dagger}+E_{01} b\right), \quad B=-i E_{00}, \\
& F=\sqrt{\gamma} b, \quad G=0, \quad W=I .
\end{aligned}
$$

This satisfies Assumptions 1 and 2 and $P_{0}=I_{\mathfrak{h}} \otimes|0\rangle\langle 0|$. Since $\left\|E_{11}\right\|<\frac{\gamma}{2}$, the inverse $\left(i E_{11}+\frac{\gamma}{2}\right)^{-1}$ exists. Let $N_{1}^{-1}: \mathcal{H}_{1} \rightarrow \mathcal{H}_{1}$ be the inverse of the restriction of $b^{\dagger} b$ to $\mathcal{H}_{1}$. 
Taking $Y_{1}^{-1}=-\left(i E_{11}+\frac{\gamma}{2}\right)^{-1} N_{1}^{-1} P_{1}$ satisfies Assumption 3. Definition 1 leads to the following coefficients

$$
\begin{aligned}
& K=-i E_{00} P_{0}-E_{01} \frac{1}{i E_{11}+\frac{\gamma}{2}} E_{10} P_{0} \\
& L=\frac{-i \sqrt{\gamma}}{i E_{11}+\frac{\gamma}{2}} E_{10} P_{0}, \quad S=\frac{i E_{11}-\frac{\gamma}{2}}{i E_{11}+\frac{\gamma}{2}} P_{0} .
\end{aligned}
$$

These coefficients satisfy Assumption 4. Theorem 2.1 then shows that $U_{t}^{(k)} P_{0}$ converges strongly to $U_{t} P_{0}$, where $U_{t}$ is given by

$$
d U_{t}=\left\{\left(S-P_{0}\right) d \Lambda_{t}+L d A_{t}^{\dagger}-L^{\dagger} S d A_{t}+K d t\right\} U_{t}, \quad U_{0}=I .
$$

Remark 1: Note that we consider a truncated oscillator, where 14 treats the full oscillator, and that we prove our result strongly, whereas 14 proves a weak limit. The convergence of the Heisenberg dynamics follows immediately from our strong result. Apart from these points, Example 3 reproduces the result in 14. Care must be taken when directly comparing the limit equations, since the results in 14 are presented in the interaction picture with respect to the cavity. Under our assumptions, we define $V_{t}^{(k)}$ as the solution to

$$
d V_{t}^{(k)}=\left\{\sqrt{\gamma} k b d A_{t}^{\dagger}-\sqrt{\gamma} k b^{\dagger} d A_{t}-\frac{\gamma k^{2}}{2} b^{\dagger} b d t\right\} V_{t}^{(k)}, \quad V_{0}^{(k)}=I .
$$

The unitary in the interaction picture is then given by $\tilde{U}_{t}^{(k)}=V_{t}^{(k) \dagger} U_{t}^{(k)}$, where $U_{t}^{(k)}$ is given by Eq. (5). Note that due to Theorem 2.1 $V_{t}^{(k)} P_{0}$ converges strongly to $V_{t} P_{0}$, where $V_{t}$ is given by

$$
d V_{t}=-2 P_{0} d \Lambda_{t} V_{t}, \quad V_{0}=I .
$$

This accounts for the sign difference between the coefficients in the equation for $\tilde{U}_{t}$ presented in [14, and the coefficients in the equation for $U_{t}$ given by Eq. (6).

Example 4: (Duan and Kimble [8]) We again consider a system inside a cavity, described by the Hilbert space $\mathcal{H}=\mathfrak{h} \otimes \mathbb{C}^{N}$. The system inside the cavity is a three level atom, i.e. $\mathfrak{h}=\mathbb{C}^{3}$. Let $(|e\rangle,|+\rangle,|-\rangle)$ be an orthogonal basis in $\mathfrak{h}$. In this basis we define

$$
\sigma_{+}^{(+)}=\left(\begin{array}{ccc}
0 & 1 & 0 \\
0 & 0 & 0 \\
0 & 0 & 0
\end{array}\right) \quad \sigma_{+}^{(-)}=\left(\begin{array}{ccc}
0 & 0 & 1 \\
0 & 0 & 0 \\
0 & 0 & 0
\end{array}\right) .
$$

Moreover define $\sigma_{-}^{( \pm)}=\sigma_{+}^{( \pm) \dagger}$ and $P_{ \pm}=\sigma_{-}^{( \pm)} \sigma_{+}^{( \pm)}$. The QSDE for a lambda system with one leg $(+\leftrightarrow e)$ resonantly coupled to the cavity, under the rotating wave approximation in the rotating frame, is,

$$
\begin{aligned}
d U_{t}^{(k)}= & \left\{\sqrt{\gamma} k b d A_{t}^{\dagger}-\sqrt{\gamma} k b^{\dagger} d A_{t}-\frac{\gamma k^{2}}{2} b^{\dagger} b d t+\right. \\
& \left.g k^{2}\left(\sigma_{+}^{(+)} b-\sigma_{-}^{(+)} b^{\dagger}\right) d t+k\left(\sigma_{+}^{(-)} \alpha-\sigma_{-}^{(-)} \bar{\alpha}\right) d t\right\} U_{t}^{(k)}, \quad U_{0}^{(k)}=I .
\end{aligned}
$$


Here $\gamma$ is a positive real parameter and $\alpha$ is a complex parameter. Note that we extend the model from [8] to allow driving on the uncoupled leg $(-\leftrightarrow e)$ of the transition. Define operators $Y, A, B, F, G, W$ as

$$
\begin{aligned}
& Y=-\frac{\gamma}{2} b^{\dagger} b+g\left(\sigma_{+}^{(+)} b-\sigma_{-}^{(+)} b^{\dagger}\right), \quad A=\left(\sigma_{+}^{(-)} \alpha-\sigma_{-}^{(-)} \bar{\alpha}\right), \quad B=0, \\
& F=\sqrt{\gamma} b, \quad G=0, \quad W=I .
\end{aligned}
$$

This satisfies Assumptions 11 and 2 and $P_{0}=(|+\rangle\langle+|+|-\rangle\langle-|) \otimes|0\rangle\langle 0|$. We define the following subspaces of $\mathcal{H}$

$$
\begin{aligned}
& H_{n}=\operatorname{span}\{|+\rangle \otimes|n\rangle,|-\rangle \otimes|n\rangle,|e\rangle \otimes|n-1\rangle\}, \quad n \in\{1, \ldots, N-1\}, \\
& H_{N}=\operatorname{span}\{|e\rangle \otimes|N-1\rangle\} .
\end{aligned}
$$

Note that $\mathcal{H}_{1}=\bigoplus_{n=1}^{N} H_{n}$ and that the subspaces $H_{n}(n \in\{1, \ldots, N\})$ are all invariant under the action of $Y$. On the subspaces $H_{n}, n \in\{1, \ldots, N-1\}, Y$ is given by

$$
\left.Y\right|_{H_{n}}=\left(\begin{array}{ccc}
-\frac{\gamma n}{2} & 0 & -g \sqrt{n} \\
0 & -\frac{\gamma n}{2} & 0 \\
g \sqrt{n} & 0 & -\frac{\gamma(n-1)}{2}
\end{array}\right),
$$

with respect to the basis $(|+\rangle \otimes|n\rangle,|-\rangle \otimes|n\rangle,|e\rangle \otimes|n-1\rangle)$. Moreover, $\left.Y\right|_{H_{N}}=-\frac{\gamma(N-1)}{2}$. The inverse is readily computed to be

$$
\left.Y\right|_{H_{n}} ^{-1}=-\frac{1}{d}\left(\begin{array}{ccc}
\frac{\gamma(n-1)}{2} & 0 & -g \sqrt{n} \\
0 & \frac{2 d}{\gamma n} & 0 \\
g \sqrt{n} & 0 & \frac{\gamma n}{2}
\end{array}\right), \quad n \in\{1, \ldots, N-1\},
$$

where $d=\frac{\gamma^{2} n(n-1)}{4}+g^{2} n$. Moreover, $\left.Y\right|_{H_{N}} ^{-1}=-\frac{2}{\gamma(N-1)}$. We now define $Y_{1}^{-1}=$ $\left.\oplus_{n=1}^{N} Y\right|_{H_{n}} ^{-1} P_{1}$. This satisfies Assumption 3, Definition 1 leads to the following coefficients

$$
K=-\frac{|\alpha|^{2} \gamma}{2 g^{2}} P_{-} \otimes|0\rangle\left\langle 0\left|, \quad L=-\frac{\gamma \alpha}{g} \sigma_{-}^{(+)} \sigma_{+}^{(-)} \otimes\right| 0\right\rangle\left\langle 0\left|, \quad S=P_{0}-2 P_{-} \otimes\right| 0\right\rangle\langle 0| .
$$

These operators satisfy Assumption 4, Theorem 2.1] then shows that $U_{t}^{(k)} P_{0}$ converges strongly to $U_{t} P_{0}$, where $U_{t}$ is given by

$$
d U_{t}=\left\{\left(S-P_{0}\right) d \Lambda_{t}+L d A_{t}^{\dagger}-L^{\dagger} S d A_{t}+K d t\right\} U_{t}, \quad U_{0}=I .
$$

Note that the ground state system is a two-level system on which $S$ acts as $\sigma_{z}$.

\section{Proof of Theorem 2.1}

Definition 2: Suppose Assumptions 1, 2, 3 and 4 hold. Let $\mathcal{B}(\mathcal{H})$ and $\mathcal{B}\left(\mathcal{H}_{0}\right)$ be the Banach spaces of all bounded operators on $\mathcal{H}$ and $\mathcal{H}_{0}$, respectively. We define for all 
$t \geq 0$ and $k \geq 0$

$$
\begin{aligned}
& T_{t}^{(k)}(X)=\mathrm{id} \otimes \phi\left(U_{t}^{\dagger} X U_{t}^{(k)}\right), \quad X \in \mathcal{B}(\mathcal{H}), \\
& T_{t}(X)=\mathrm{id} \otimes \phi\left(U_{t}^{\dagger} X U_{t}\right), \quad X \in \mathcal{B}\left(\mathcal{H}_{0}\right),
\end{aligned}
$$

where $U_{t}^{(k)}$ and $U_{t}$ are given by Eqs. (11) and (41), respectively.

Note that $T_{t}^{(k)}$ is intentionally skew with respect to $U_{t}$ and $U_{t}^{(k)}$.

Lemma 2: For each $k>0$, the families of bounded linear maps $T_{t}^{(k)}(t \geq 0)$ and $T_{t}(t \geq$ $0)$ given by Definition 2 are norm continuous one-parameter contraction semigroups with generators

$$
\begin{aligned}
& \mathscr{L}^{(k)}(X)=K^{\dagger} X+X K^{(k)}+L_{i}^{\dagger} X L_{i}^{(k)}, \quad X \in \mathcal{B}(\mathcal{H}), \\
& \mathscr{L}(X)=K^{\dagger} X+X K+L_{i}^{\dagger} X L_{i}, \quad X \in \mathcal{B}\left(\mathcal{H}_{0}\right),
\end{aligned}
$$

respectively. That is $T_{t}^{(k)}=\exp \left(t \mathscr{L}^{(k)}\right)$ and $T_{t}=\exp (t \mathscr{L})$ for all $t \geq 0$.

Proof. We only prove the lemma for $T_{t}^{(k)}$. The proof for $T_{t}$ can be obtained in an analogous way. Since the conditional expectation id $\otimes \phi$ is norm contractive and $U_{t}$ and $U_{t}^{(k)}$ are unitary, we have

$$
\left\|T_{t}^{(k)}(X)\right\| \leq\left\|U_{t}^{\dagger} X U_{t}^{(k)}\right\| \leq\left\|U_{t}^{\dagger}\right\|\left\|U_{t}^{(k)}\right\|\|X\|=\|X\|,
$$

for all $X \in \mathcal{B}(\mathcal{H})$. This proves that $T_{t}^{(k)}$ is a contraction for all $t \geq 0$. An application of the quantum Itô rule [15, together with the fact that vacuum expectations of stochastic integrals vanish, shows that

$$
\begin{aligned}
& d T_{t}^{(k)}(X)=\mathrm{id} \otimes \phi\left(d\left(U_{t}^{\dagger} X U_{t}^{(k)}\right)\right)= \\
& \operatorname{id} \otimes \phi\left(U_{t}^{\dagger}\left(K^{\dagger} X+X K^{(k)}+L_{i}^{\dagger} X L_{i}^{(k)}\right) U_{t}^{(k)}\right) d t=T_{t}^{(k)}\left(\mathscr{L}^{(k)}(X)\right) d t,
\end{aligned}
$$

for all $X \in \mathcal{B}(\mathcal{H})$. That is, $T_{t}^{(k)}=\exp \left(t \mathscr{L}^{(k)}\right)$ is a one-parameter semigroup with generator $\mathscr{L}^{(k)}$. Furthermore, $\mathscr{L}^{(k)}$ is bounded

$$
\left\|\mathscr{L}^{(k)}(X)\right\| \leq\left(\left\|K^{\dagger}\right\|+\left\|K^{(k)}\right\|+\left\|L_{i}^{\dagger}\right\|\left\|L_{i}^{(k)}\right\|\right)\|X\|,
$$

which proves that $T_{t}^{(k)}$ is norm continuous.

The proof of Theorem 2.1] relies heavily on the Trotter-Kato theorem [21, 16] in combination with an argument due to Kurtz [17. We have taken the formulation of the Trotter-Kato theorem from [3, Thm 3.17, page 80], see also [10, Chapter 1, Section 6]. The formulation is more general than needed for the proof of Theorem 2.1 . 
Theorem 4.1: Trotter-Kato Theorem Let $\mathcal{B}$ be a Banach space and let $\mathcal{B}_{0}$ be a closed subspace of $\mathcal{B}$. For each $k \geq 0$, let $T_{t}^{(k)}$ be a strongly continuous one-parameter contraction semigroup on $\mathcal{B}$ with generator $\mathscr{L}^{(k)}$. Moreover, let $T_{t}$ be a strongly continuous one-parameter contraction semigroup on $\mathcal{B}_{0}$ with generator $\mathscr{L}$. Let $\mathcal{D}$ be a core for $\mathscr{L}$. The following conditions are equivalent:

1. For all $X \in \mathcal{D}$ there exist $X^{(k)} \in \operatorname{Dom}\left(\mathscr{L}^{(k)}\right)$ such that

$$
\lim _{k \rightarrow \infty} X^{(k)}=X, \quad \lim _{k \rightarrow \infty} \mathscr{L}^{(k)}\left(X^{(k)}\right)=\mathscr{L}(X) .
$$

2. For all $0 \leq s<\infty$ and all $X \in \mathcal{B}_{0}$

$$
\lim _{k \rightarrow \infty}\left\{\sup _{0 \leq t \leq s}\left\|T_{t}^{(k)}(X)-T_{t}(X)\right\|\right\}=0 .
$$

Proposition 1: Let $T_{t}^{(k)}$ and $T_{t}$ be the one-parameter semigroups on $\mathcal{B}(\mathcal{H})$ and $\mathcal{B}\left(\mathcal{H}_{0}\right)$ defined in Definition 2, respectively. We have

$$
\lim _{k \rightarrow \infty}\left\{\sup _{0 \leq t \leq s}\left\|T_{t}^{(k)}(X)-T_{t}(X)\right\|\right\}=0,
$$

for all $X \in \mathcal{B}\left(\mathcal{H}_{0}\right)$ and $0 \leq s<\infty$.

Proof. The proof follows the line of the proof of [17, Theorem 2.2]. Lemma 2 shows that $T_{t}^{(k)}=\exp \left(t \mathscr{L}^{(k)}\right)$ and $T_{t}=\exp (t \mathscr{L})$ are norm continuous, and therefore also strongly continuous semigroups with generators given by Eq. (7). This means we satisfy the assumptions of the Trotter-Kato Theorem (Thm. 4.1) with $\mathcal{D}=\mathcal{B}\left(\mathcal{H}_{0}\right)$ and $\operatorname{Dom}\left(\mathscr{L}^{(k)}\right)=\mathcal{B}(\mathcal{H})$.

We can write $\mathscr{L}^{(k)}(X)=\mathscr{L}_{0}(X)+k \mathscr{L}_{1}(X)+k^{2} \mathscr{L}_{2}(X), \quad X \in \mathcal{B}(\mathcal{H})$, where (recall Assumption 2)

$$
\mathscr{L}_{0}(X)=K^{\dagger} X+X B+L_{i}^{\dagger} X G_{i}, \quad \mathscr{L}_{1}(X)=X A+L_{i}^{\dagger} X F_{i}, \quad \mathscr{L}_{2}(X)=X Y .
$$

Let $X$ be an element in $\mathcal{B}\left(\mathcal{H}_{0}\right)$ and let $X_{1}$ and $X_{2}$ be elements in $\mathcal{B}(\mathcal{H})$. We define $X^{(k)}=X+\frac{1}{k} X_{1}+\frac{1}{k^{2}} X_{2}$. Collecting terms with equal powers in $k$, we find

$$
\begin{aligned}
\mathscr{L}^{(k)}\left(X^{(k)}\right)= & \left(\mathscr{L}_{0}(X)+\mathscr{L}_{1}\left(X_{1}\right)+\mathscr{L}_{2}\left(X_{2}\right)\right)+ \\
& k\left(\mathscr{L}_{1}(X)+\mathscr{L}_{2}\left(X_{1}\right)\right)+ \\
& k^{2}\left(\mathscr{L}_{2}(X)\right)+ \\
& \frac{1}{k}\left(\mathscr{L}_{0}\left(X_{1}\right)+\mathscr{L}_{1}\left(X_{2}\right)\right)+\frac{1}{k^{2}}\left(\mathscr{L}_{0}\left(X_{2}\right)\right) .
\end{aligned}
$$

Note that $\mathscr{L}_{2}(X)=0$ as $X \in \mathcal{B}\left(\mathcal{H}_{0}\right)$ and $P_{0} Y=0$. Using the existence of $Y_{1}^{-1}$, we set

$$
\begin{aligned}
& X_{1}=-\mathscr{L}_{1}(X) Y_{1}^{-1} P_{1}, \\
& X_{2}=-\left(\mathscr{L}_{0}(X)+\mathscr{L}_{1}\left(X_{1}\right)\right) Y_{1}^{-1} P_{1} .
\end{aligned}
$$


Using the properties of $Y_{1}^{-1}$ in Assumption 3, we obtain

$$
\begin{aligned}
\lim _{k \rightarrow \infty} \mathscr{L}^{(k)}\left(X^{(k)}\right) & =\lim _{k \rightarrow \infty}\left(\mathscr{L}(X)+\frac{1}{k}\left(\mathscr{L}_{0}\left(X_{1}\right)+\mathscr{L}_{1}\left(X_{2}\right)\right)+\frac{1}{k^{2}} \mathscr{L}_{0}\left(X_{2}\right)\right) \\
& =\mathscr{L}(X) .
\end{aligned}
$$

The proposition then follows from the Trotter-Kato Theorem.

Note that for all $v \in \mathcal{H}_{0}$, we can write $U_{t} v \otimes \Phi=P_{0} U_{t} v \otimes \Phi$. This leads to

$$
\begin{aligned}
\left\|\left(U_{t}^{(k)}-U_{t}\right) v \otimes \Phi\right\|^{2} & =\left\|\left(U_{t}^{(k)}-P_{0} U_{t}\right) v \otimes \Phi\right\|^{2} \\
& =\left\langle v,\left(2 I-T_{t}^{(k)}\left(P_{0}\right)-T_{t}^{(k)}\left(P_{0}\right)^{\dagger}\right) v\right\rangle .
\end{aligned}
$$

Here we have used that id $\otimes \phi$ is a positive map, i.e. it commutes with the adjoint. Using Proposition 1 and noting that $\mathscr{L}\left(P_{0}\right)=0$ by Lemmas 2 and 1 , we see that Theorem 2.1 holds for all vectors in $\mathcal{H}_{0} \otimes \mathcal{F}$ of the form $\psi=v \otimes \Phi$. We now need to extend this to all $\psi \in \mathcal{H}_{0} \otimes \mathcal{F}$.

Let $f$ be an element in $L^{2}\left(\mathbb{R}^{+} ; \mathbb{C}^{n}\right)$. Denote by $f_{t}$ the function $f$ truncated at time $t$, i.e. $f_{t}(s)=f(s)$ if $s \leq t$ and $f_{t}(s)=0$ otherwise. Define the Weyl operator $W\left(f_{t}\right)$ as the unique solution to the following QSDE

$$
d W\left(f_{t}\right)=\left\{f(t)_{i} d A_{t}^{i \dagger}-\overline{f(t)}_{i} d A_{t}^{i}-\frac{1}{2} \overline{f(t)}{ }_{i} f(t)_{i} d t\right\} W\left(f_{t}\right), \quad W\left(f_{0}\right)=I .
$$

Note that $W\left(f_{t}\right)$ is a unitary operator from $\mathcal{F}$ to $\mathcal{F}$. Moreover, it is not hard to see that $\pi\left(f_{t}\right)=W\left(f_{t}\right) \Phi$, see e.g. [20]. Often we will identify a constant $\alpha \in \mathbb{C}^{n}$ with the constant function on $\mathbb{R}^{+}$taking the value $\alpha$ (truncated at some large $T \geq 0$ so that it is an element of $\left.L^{2}\left(\mathbb{R}^{+} ; \mathbb{C}^{n}\right)\right)$.

Definition 3: Let $f$ be an element in $L^{2}\left(\mathbb{R}^{+} ; \mathbb{C}^{n}\right)$. Suppose that Assumptions 11, 2, 3 and 4 hold and let $U_{t}^{(k)}$ and $U_{t}$ be given by Eqs. (1) and (4), respectively. Define

$$
\begin{aligned}
& U_{t}^{(k f)}=W\left(f_{t}\right)^{\dagger} U_{t}^{(k)} W\left(f_{t}\right), \quad U_{t}^{(f)}=W\left(f_{t}\right)^{\dagger} U_{t} W\left(f_{t}\right), \\
& T_{t}^{(k f)}(X)=\operatorname{id} \otimes \phi\left(U_{t}^{(f) \dagger} X U_{t}^{(k f)}\right), \quad X \in \mathcal{B}(\mathcal{H}), \\
& T_{t}^{(k f)}(X)=\operatorname{id} \otimes \phi\left(U_{t}^{(f) \dagger} X U_{t}^{(f)}\right), \quad X \in \mathcal{B}\left(\mathcal{H}_{0}\right) .
\end{aligned}
$$

Definition 4: Let $\alpha$ be an element in $\mathbb{C}^{n}$ and let $i$ be an element in $\{1, \ldots, n\}$. Let $K^{(k)}, K, L_{i}^{(k)}, L_{i}, S_{i j}^{(k)}$ and $S_{i j}$ be the coefficients of Eqs. (11) and (4). Define operators $K^{(k \alpha)}, K^{(\alpha)}, L_{i}^{(k \alpha)}$ and $L_{i}^{(\alpha)}$ by

$$
\begin{aligned}
& K^{(\alpha)}=K+\bar{\alpha}_{i}\left(S_{i j}-P_{0} \delta_{i j}\right) \alpha_{j}+\bar{\alpha}_{i} L_{i}-\alpha_{j} L_{i}^{\dagger} S_{i j}, \quad L_{i}^{(\alpha)}=L_{i}+\alpha_{j} S_{i j}, \\
& K^{(k \alpha)}=K^{(k)}+\bar{\alpha}_{i}\left(S_{i j}^{(k)}-\delta_{i j}\right) \alpha_{j}+\bar{\alpha}_{i} L_{i}^{(k)}-\alpha_{j} L_{i}^{(k) \dagger} S_{i j}, \quad L_{i}^{(k \alpha)}=L_{i}^{(k)}+\alpha_{j} S_{i j}^{(k)} .
\end{aligned}
$$


Note that with the coefficients given by Definition 4, applying the quantum Itô rule to $U_{t}^{(k \alpha)}$ and $U_{t}^{(\alpha)}$, defined in Definition 3 gives

$$
\begin{aligned}
& d U_{t}^{(\alpha)}=\left\{\left(S_{i j}-\delta_{i j} P_{0}\right) d \Lambda_{t}^{i j}+L_{i}^{(\alpha)} d A_{t}^{i \dagger}-L_{i}^{(\alpha) \dagger} S_{i j} d A_{t}^{j}+K^{(\alpha)} d t\right\} U_{t}^{(\alpha)}, \\
& d U_{t}^{(k \alpha)}=\left\{\left(S_{i j}^{(k)}-\delta_{i j}\right) d \Lambda_{t}^{i j}+L_{i}^{(k \alpha)} d A_{t}^{i \dagger}-L_{i}^{(k \alpha) \dagger} S_{i j}^{(k)} d A_{t}^{j}+K^{(k \alpha)} d t\right\} U_{t}^{(k \alpha)},
\end{aligned}
$$

with $U_{0}^{(\alpha)}=U_{0}^{(k \alpha)}=I$.

Definition 5: Suppose that Assumptions 1, 2, 3 and 4hold. Let $\alpha$ be an element in $\mathbb{C}^{n}$ and let $i$ be an element in $\{1, \ldots, n\}$. Define operators $A^{(\alpha)}, B^{(\alpha)}$ and $G_{i}^{(\alpha)}$ by

$$
\begin{aligned}
& A^{(\alpha)}=A+F_{i} \bar{\alpha}_{i}-\alpha_{j} F_{i}^{\dagger} W_{i j}, \\
& B^{(\alpha)}=B+\bar{\alpha}_{i}\left(W_{i j}-\delta_{i j}\right) \alpha_{j}+G_{i} \bar{\alpha}_{i}-\alpha_{j} G_{i}^{\dagger} W_{i j}, \\
& G_{i}^{(\alpha)}=G_{i}+\alpha_{j} W_{i j} .
\end{aligned}
$$

Lemma 3: Suppose Assumptions 1, 2, 3] and 4 hold. Let $A, B, Y, F_{i}, G_{i}, W_{i j}, K, L_{i}$ and $S_{i j}$ for $i, j \in\{1, \ldots, n\}$ be the various operators occuring in Assumption [1, 2, 3 and 4 Let $K^{(\alpha)}$ and $L_{i}^{(\alpha)}$ for $i \in\{1, \ldots, n\}$ be given by Definition 4 and let $A^{(\alpha)}, B^{(\alpha)}$ and $G_{i}^{(\alpha)}$ for $i \in\{1, \ldots, n\}$ be given by Definition 5 , Then

$$
\begin{aligned}
& L_{i}^{(\alpha)}=\left(G_{i}^{(\alpha)}-F_{i} Y_{1}^{-1} A^{(\alpha)}\right) P_{0}, \\
& K^{(\alpha)}=P_{0}\left(B^{(\alpha)}-A^{(\alpha)} Y_{1}^{-1} A^{(\alpha)}\right) P_{0},
\end{aligned}
$$

i.e. Definition 1 holds with $A=A^{(\alpha)}, B=B^{(\alpha)}, G_{i}=G_{i}^{(\alpha)} L_{i}=L_{i}^{(\alpha)}$ and $K=K^{(\alpha)}$. Moreover, Assumptions 1, 2, 3] and [4 hold for the altered coefficients with $P_{0}$ and $Y_{1}^{-1}$ unchanged.

Proof. To show that Definition 1 holds for the altered coefficients, substitute $G_{i}^{(\alpha)}$ and $A^{(\alpha)}$ from Definition [5, and $L_{i}^{(\alpha)}$ from Definition 4 into Eq. (10a). This gives

$$
L_{i}+\alpha_{j} S_{i j}=\left(L_{i}+\alpha_{j} W_{i j}+\alpha_{j} F_{i} Y_{1}^{-1} F_{l}^{\dagger} W_{l j},\right) P_{0},
$$

which holds if we substitute $S_{i j}=\left(W_{i j}+F_{i} Y_{1}^{-1} F_{l}^{\dagger} W_{l j}\right) P_{0}$ from Definition 1 Furthermore, substituting $A^{(\alpha)}$ and $B^{(\alpha)}$ from Definition [5, and $K^{(\alpha)}$ from Definition 4 into Eq. (10b) gives

$$
\begin{aligned}
& \bar{\alpha}_{i} S_{i j} \alpha_{j}+\bar{\alpha}_{i} L_{i}-\alpha_{j} L_{i}^{\dagger} S_{i j}=P_{0} \bar{\alpha}_{i} W_{i j} \alpha_{j} P_{0}+P_{0} G_{i} P_{0} \bar{\alpha}_{i}-\alpha_{j} P_{0} G_{i}^{\dagger} W_{i j} P_{0} \\
& -P_{0}\left(F_{i} \bar{\alpha}_{i}+A\right) Y_{1}^{-1}\left(A-\alpha_{j} F_{l}^{\dagger} W_{l j}\right) P_{0}+P_{0} A Y_{1}^{-1} A P_{0} .
\end{aligned}
$$


This holds if we can show that

$$
\begin{aligned}
& S_{i j}=P_{0}\left(W_{i j}+F_{i} Y_{1}^{-1} F_{l}^{\dagger} W_{l j}\right) P_{0} \\
& L_{i}=P_{0}\left(G_{i}-F_{i} Y_{1}^{-1} A\right) P_{0} \\
& L_{i}^{\dagger} S_{i j}=P_{0}\left(G_{i}^{\dagger} W_{i j}-A Y_{1}^{-1} F_{i}^{\dagger} W_{i j}\right) P_{0} .
\end{aligned}
$$

Equations (11a) and (11b) are satisfied by Assumption 4 as $P_{1} L_{i}=P_{1} S_{i j}=0$. Note that Eq. (11c) holds if we can show

$$
L_{i}^{\dagger}\left(\delta_{i l}+F_{i} Y_{1}^{-1} F_{l}^{\dagger}\right) W_{l j} P_{0}=P_{0} G_{l}^{\dagger} W_{l j} P_{0}-P_{0} A Y_{1}^{-1} F_{l}^{\dagger} W_{l j} P_{0} .
$$

Substituting $L_{i}$ from Definition [1, this becomes

$$
\begin{aligned}
-P_{0} A^{\dagger} Y_{1}^{-1 \dagger} F_{l}^{\dagger} W_{l j} P_{0}+ & P_{0} G_{i}^{\dagger} F_{i} Y_{1}^{-1} F_{l}^{\dagger} W_{l j} P_{0} \\
& \quad-P_{0} A^{\dagger} Y_{1}^{-1 \dagger} F_{i}^{\dagger} F_{i} Y_{1}^{-1} F_{l}^{\dagger} W_{l j} P_{0}+P_{0} A Y_{1}^{-1} F_{l}^{\dagger} W_{l j} P_{0}=0 .
\end{aligned}
$$

Now recall that $P_{0}\left(A+A^{\dagger}\right) P_{1}=-P_{0} G_{i}^{\dagger} F_{i} P_{1}$, and $Y+Y^{\dagger}=-F_{i}^{\dagger} F_{i}$ (see Eq. (3)) by Assumptions 1, 22 and 3. Moreover, $Y Y_{1}^{-1} P_{1} F_{l}^{\dagger} W_{l j} P_{0}=P_{1} F_{l}^{\dagger} W_{l j} P_{0}$ by Assumption 3 which shows that Eq. (11c) is satisfied.

We now show that Assumptions 1, 2, 3 and 4 hold for the altered coefficients, with $P_{0}$ and $Y_{1}^{-1}$ unchanged. Assumption 1 holds for the altered coefficients since, by Definition 3. we have $U_{t}^{(k \alpha)}=W\left(f_{t}\right)^{\dagger} U_{t}^{(k)} W\left(f_{t}\right)$ which is clearly unitary. By Assumption 2 for the original coefficients and Definition 4 and [5] we see that Assumption 2 holds for the altered coefficients. Assumption 3 on the altered coefficients is seen to hold by direct substitution of the coefficients in Definition 4 and 5. followed by application of Assumption 3 for the original system. Assumption 4 holds if $P_{1} L_{i}^{(\alpha)}=P_{1} L_{i}+\alpha_{i} P_{1} S_{i j}=0$, which follows from Assumption 4 on the original system.

Lemma 3 shows that Proposition 1 holds with $T_{t}^{(k \alpha)}$ and $T_{t}^{(\alpha)}$ replacing $T_{t}^{(k)}$ and $T_{t}$, respectively.

Corollary 1: Suppose that Assumption 1, 2, 3 and 4 hold. Let $\alpha$ be an element of $\mathbb{C}^{n}$. We have

$$
\lim _{k \rightarrow \infty}\left\{\sup _{0 \leq t \leq s}\left\|T_{t}^{(k \alpha)}(X)-T_{t}^{(\alpha)}(X)\right\|\right\}=0,
$$

for all $X \in \mathcal{B}\left(\mathcal{H}_{0}\right)$ and $0 \leq s<\infty$.

Proof of Theorem 2.1. Let $t \geq 0$. Let $f$ be a step function in $L^{2}\left([0, t] ; \mathbb{C}^{n}\right)$, i.e. there exists an $m \in \mathbb{N}$ and $0=t_{0}<t_{1}<\ldots<t_{m}=t$ and $\alpha_{1}, \ldots, \alpha_{m} \in \mathbb{C}^{n}$ such that

$$
s \in\left[t_{i-1}, t_{i}\right) \Longrightarrow f(s)=\alpha_{i}, \quad \forall i \in\{1, \ldots, m\} .
$$


The cocycle property of solutions to QSDE's and the exponential property of the symmetric Fock space lead to

$$
\begin{aligned}
& T_{t}^{(k f)}(X)=T_{t_{1}}^{\left(k \alpha_{m}\right)} \ldots T_{t-t_{m-1}}^{\left(k \alpha_{1}\right)}(X), \quad X \in \mathcal{B}(\mathcal{H}), \\
& T_{t}^{(f)}(X)=T_{t_{1}}^{\left(\alpha_{m}\right)} \ldots T_{t-t_{m-1}}^{\left(\alpha_{1}\right)}(X), \quad X \in \mathcal{B}\left(\mathcal{H}_{0}\right) .
\end{aligned}
$$

It is easy to see that Corollary 1 also holds for the difference of a finite product of maps $T_{t_{i}-t_{i-1}}^{\left(k \alpha_{i}\right)}$ and a finite product of maps $T_{t_{i}-t_{i-1}}^{\left(\alpha_{i}\right)}$. This leads to

$$
\begin{aligned}
& \lim _{k \rightarrow \infty}\left\|T_{t}^{(k f)}(X)-T_{t}^{(f)}(X)\right\|= \\
& \lim _{k \rightarrow \infty}\left\|T_{t_{1}}^{\left(k \alpha_{m}\right)} \ldots T_{t-t_{m-1}}^{\left(k \alpha_{1}\right)}(X)-T_{t_{1}}^{\left(\alpha_{m}\right)} \ldots T_{t-t_{m-1}}^{\left(\alpha_{1}\right)}(X)\right\|=0, \quad X \in \mathcal{B}\left(\mathcal{H}_{0}\right) .
\end{aligned}
$$

This immediately yields for all step functions $f \in L^{2}\left([0, t] ; \mathbb{C}^{n}\right)$ and $v \in \mathcal{H}_{0}$

$$
\lim _{k \rightarrow \infty} U_{t}^{(k)} v \otimes \pi(f)=U_{t} v \otimes \pi(f) .
$$

Note that the step functions are dense in $L^{2}\left([0, t] ; \mathbb{C}^{n}\right)$. This means that Eq. (12) holds for all $f \in L^{2}\left([0, t] ; \mathbb{C}^{n}\right)$. Now note that for all $f \in L^{2}\left(\mathbb{R}^{+} ; \mathbb{C}^{n}\right)$ and $t \leq s \leq \infty$, we have (e.g. [20])

$$
W\left(f_{s}\right)^{\dagger} U_{t}^{(k)} W\left(f_{s}\right)=U_{t}^{\left(k f_{t}\right)}, \quad W\left(f_{s}\right)^{\dagger} U_{t} W\left(f_{s}\right)=U_{t}^{\left(f_{t}\right)} .
$$

This means that the result in Eq. (12) is true for all $f \in L^{2}\left(\mathbb{R}^{+} ; \mathbb{C}^{n}\right)$. We now have

$$
\lim _{k \rightarrow \infty} U_{t}^{(k)} \psi=U_{t} \psi
$$

for all $\psi$ in $\mathscr{D}=\operatorname{span}\left\{v \otimes \pi(f) ; v \in \mathcal{H}_{0}, f \in L^{2}\left(\mathbb{R}^{+} ; \mathbb{C}^{n}\right)\right\}$. Theorem 2.1 then follows from the fact that $\mathscr{D}$ is dense in $\mathcal{H}_{0} \otimes \mathcal{F}$ (e.g. [20]).

\section{Discussion}

In this article we have studied adiabatic elimination in the context of the quantum stochastic models introduced by Hudson and Parthasarathy. We have shown strong convergence of a quantum stochastic differential equation to its adiabatically eliminated counterpart, under four assumptions. Physically, the first Assumption 1 enforces the unitarity of the initial QSDE model. Assumption 2 ensures an appropriate scaling in the coupling parameter $k$ such that we can distinguish excited and ground states in our system. Assumptions 3 and 4 ensure the existence of a limit dynamics independent of $k$. Note that Assumption 4 specifically forbids any quantum jumps which terminate in an excited state, the presence of which would preclude the construction of a valid limit dynamics.

Although a Dyson series expansion for $U_{t}^{(k)}$ (e.g. in terms of Maassen kernels [18]) would provide a lot of intuition for the results we have obtained (see [14] and 3, Chapter 5, Section 4]), we have chosen a proof along the lines of semigroups and their generators. 
An infinitesimal treatment has the advantage that it can exploit the existence of results such as the quantum Itô rule [15, the Trotter-Kato Theorem [21, 16] and the technique due to Kurtz [17].

Acknowledgement. We thank Mike Armen, Ramon van Handel and Hideo Mabuchi for stimulating discussion. We especially thank Ramon van Handel for pointing out mistakes in an earlier version of this work. L.B. is supported by the ARO under Grant No. W911NF-06-1-0378. A.S. acknowledges support by the ONR under Grant No. N0001405-1-0420.

\section{References}

[1] L. Accardi, A. Frigerio, and Y. Lu. The weak coupling limit as a quantum functional central limit. Commun. Math. Phys., 131:537-570, 1990.

[2] C. Cohen-Tannoudji, J. Dupont-Roc, and G. Grynberg. Atom-Photon Interactions: Basic Processes and Applications. John Wiley \& Sons, 1992.

[3] E. Davies. One-parameter semigroups. Academic Press Inc (London) Ltd, 1980.

[4] E. B. Davies. Particle-boson interactions and the weak coupling limit. J. Math. Phys., 20:345-351, 1979.

[5] J. Derezinski and W. De Roeck. Extended weak coupling limit for Pauli-Fierz operators, 2006. math-ph/0610054.

[6] I. Deutsch and P. Jessen. Optical lattices. Advances in Atomic, Molecular and Optical Physics, 37:95, 1996.

[7] A. Doherty, A. Parkins, S. Tan, and D. Walls. Motional states of atoms in cavity qed. Phys. Rev. A, 47:4804-4817, 1998.

[8] L.-M. Duan and H. Kimble. Scalable photonic quantum computation through cavityassisted interaction. Phys. Rev. Lett., 92:127902, 2004.

[9] J. Dunningham, H. Wiseman, and D. Walls. Manipulating the motion of a single atom in a standing wave via feedback. Phys. Rev. A, 55:1398-1411, 1997.

[10] S. Ethier and T. Kurtz. Markov Processes: Characterization and Convergence. John Wiley \& Sons, Inc., 1986.

[11] C. Gardiner. Adiabatic elimination in stochastic systems. i. formulation of methods and application to few variable systems. Phys. Rev. A, 29:2814-2822, 1984.

[12] C. Gardiner and P. Zoller. Quantum Noise. Springer, Berlin, 2000.

[13] J. Gough. Quantum flows as Markovian limit of emission, absorption and scattering interactions. Commun. Math. Phys., 254:489-512, 2005.

[14] J. Gough and R. van Handel. Singular perturbation of quantum stochastic differential equations with coupling through an oscillator mode. J. Stat. Phys., 127:575, 2007. 
[15] R. L. Hudson and K. R. Parthasarathy. Quantum Itô's formula and stochastic evolutions. Commun. Math. Phys., 93:301-323, 1984.

[16] T. Kato. Remarks on pseudo-resolvents and infinitesimal generators of semigroups. Proc. Japan. Acad., 35:467-468, 1959.

[17] T. Kurtz. A limit theorem for perturbed operator semigroups with applications to random evolutions. J. Funct. Anal., 12:55-67, 1973.

[18] H. Maassen. Quantum Markov processes on Fock space described by integral kernels. In L. Accardi and W. von Waldenfels, editors, QP and Applications II, volume 1136 of Lecture Notes in Mathematics, pages 361-374. Springer, Berlin, 1985.

[19] G. Papanicolaou. Some probabilistic problems and methods in singular perturbations. Rocky Mnt. J. Math, 6:653-674, 1976.

[20] K. R. Parthasarathy. An Introduction to Quantum Stochastic Calculus. Birkhäuser, Basel, 1992.

[21] H. Trotter. Approximations of semigroups of operators. Pacific J. Math., 8:887-919, 1958.

[22] D. Walls and G. Milburn. Quantum Optics. Springer Verlag, Berlin Heidelberg, 1994.

[23] H. M. Wiseman and G. J. Milburn. Quantum theory of field-quadrature measurements. Phys. Rev. A, 47:642-662, 1993.

[24] K. Wong, M. Collett, and D. Walls. Atomic juggling using feedback. Opt. Commun., 137:269-275, 1997. 